\title{
Using telemedicine to screen for retinopathy of prematurity
}

\author{
Ayman A. Abdul Aziz MD, Maram Isaac MD MHA, Nasrin Najm Tehrani MB BCh
}

Competing interests: None declared.

This article has been peer reviewed.

Correspondence to:

Nasrin Najm Tehrani, nasrin.tehrani@sickkids.ca

CMAJ 2014. DOI:10.1503 /cmaj.131357
$\mathrm{T}$ elemedicine is increasingly being used in ophthalmology for diagnosis, education and research. Combined with digital imaging, telemedicine has been successful in screening for diabetic retinopathy and diagnosing macular diseases and glaucoma. ${ }^{1}$ The introduction of the digital pediatric fundus camera has enabled the use of telemedicine in the detection and monitoring of a variety of pediatric eye diseases, including retinopathy of prematurity, retinoblastoma and nonaccidental trauma. ${ }^{1}$

Retinopathy of prematurity is a vascular proliferative disease of the retina that can result in severe complications leading to blindness in preterm infants. Binocular indirect ophthalmoscopy is the gold standard for eye examinations. Timing of the examinations is critical, and they are usually started while the infant is in the neonatal intensive care unit (ICU). The Canadian Paediatric Society has developed guidelines to screen infants for retinopathy of prematurity. ${ }^{2}$ Timely treatment substantially reduces the occurrence of complications and the risk of blindness. ${ }^{3}$

Limited access to appropriately timed eye examinations may result from a shortage of experienced ophthalmologists trained to screen and treat retinopathy of prematurity. At-risk infants in remote areas are sometimes transferred to a central (level III) neonatal ICU for each examination. Transfers of infants from

\footnotetext{
- Ker POINTS

- Timely screening for and treatment of retinopathy of prematurity reduce the risk of blindness in infants.

- The level of access to experienced ophthalmologic care is suboptimal in remote areas.

- The combination of telemedicine and digital imaging using a pediatric fundus camera may improve access to screening services.

- A real-time audio-video connection allows optimal supervision of imaging personnel and connects ophthalmologists directly with parents.
}

level III nurseries to more remote ones are often postponed to ensure the eye examinations take place. Both options incur substantial health care costs, are associated with increased morbidity and are inconvenient for families.

Each year, more than 4000 premature infants in Canada undergo multiple eye examinations for the screening of retinopathy of prematurity. Fewer than 150 of them are found to have severe retinopathy requiring treatment. ${ }^{4}$

\section{How are digital imaging and telemedicine combined to screen for retinopathy of prematurity?}

The addition of the pediatric fundus camera as a telemedicine tool brings expertise from central neonatal ICUs to the bedside in remote units. Imaging of the infant retina is performed differently from imaging of the adult retina. The pediatric fundus camera is a wide-angle digital camera that is placed in contact with the eye to allow photography of the peripheral retina. RetCam (Clarity Medical Systems, Pleasanton, Calif.) is the most commonly used system. At the remote site, neonatal personnel trained in the use of the camera take images of the infant's retinas. The images are then uploaded to a secure server at a central reading centre. An ophthalmologist at the reading centre evaluates the images using software that facilitates detailed image analysis. A final report and a management plan are sent back to the remote unit. This system uses a store-andforward technique to share images..$^{5-7}$

An alternative to the store-and-forward system is the use of a real-time, two-way audio-video connection. The ophthalmologist in the reading centre and personnel in the remote neonatal ICU communicate directly during the imaging session using mobile video-teleconferencing devices on a secure Internet-based connection. The video camera of the teleconferencing unit at the remote site 
is positioned to allow direct guidance from the central reading site. Fine adjustment of light and focusing parameters is critical in obtaining clear images for safe evaluation of the severity of the disease. (A video showing the use of telemedicine and digital imaging to screen an infant for retinopathy of prematurity is available in Appendix 1, www.cmaj.ca/lookup/suppl/doi:10.1503 /cmaj.131357/-/DC1).

The acquisition of high-quality images with a pediatric fundus camera by personnel without prior expertise in contact ophthalmic imaging is a skill that takes time to master and may require prolonged support from experienced specialists. ${ }^{8}$ Data from two telemedicine screening programs for retinopathy of prematurity ${ }^{5,6}$ suggest the need for repeat imaging, particularly in younger infants. ${ }^{5}$ Because each screening examination may have associated systemic and ocular morbidities (e.g., changes in blood pressure, desaturation episodes and retinal hemorrhage), ${ }^{9}$ eliminating unnecessary imaging sessions is important for the safety of these fragile infants. The real-time video connection provides opportunities for continuing education and supervision of personnel performing the screening examinations at the remote unit to obtain imaging of optimal quality while maintaining patient safety. In addition, it facilitates direct parentphysician communication.

The Ontario Telemedicine for Retinopathy of Prematurity (ONTROP) project is a pilot program started in 2009 that uses real-time video communication. This project is described in Appendix 2 (available at www.cmaj.ca/lookup /suppl/doi:10.1503/cmaj.131357/-/DC1).

\section{Who will benefit from this application of telemedicine?}

Telemedicine and digital imaging can potentially provide access to resources for screening preterm infants in underserved neonatal ICUs located far from major treatment centres., ${ }^{5,6}$ The number of infants who need to be screened has increased, whereas the number of ophthalmologists willing to screen and treat retinopathy of prematurity has decreased. The reasons for this decrease include limited reimbursement, time constraints and liability risks, ${ }^{5,10}$ as well as scope of practice limited to adults and a lack of training or experience in screening and treating the disease..$^{10}$ Many screening programs have been developed to use telemedicine and digital imaging. ${ }^{5.6,11}$ One example is the current model being used in India, where an estimated $65000-$ 130000 infants require screening for retinopathy of prematurity each year. ${ }^{11}$ The program con- sists of 18 rural and semiurban centres where technicians have been trained to use a portable digital fundus camera and a store-and-forward imaging system. ${ }^{11}$

\section{What is the evidence so far?}

A systematic review of screening strategies using digital imaging for retinopathy of prematurity concluded that the current evidence base is not sufficient to recommend the routine use of retinal imaging by neonatal units to identify infants who have severe retinopathy. ${ }^{12}$ However, multiple community-based studies reported positively on the potential for telemedicine screening in remote areas to detect disease that will eventually require treatment..$^{5-7}$ In a retrospective analysis of the first four years of its telemedicine initiative to screen for retinopathy of prematurity, the Stanford University Network for Diagnosis of Retinopathy of Prematurity reported that none of the infants who needed treatment were missed. ${ }^{5}$ Weaver and Murdock $^{6}$ reported that a telemedicine screening program in Montana had $100 \%$ sensitivity in the detection of infants with retinopathy of prematurity who eventually required treatment. Similarly, the authors of a multicentre study in Germany that involved five peripheral neonatal ICUs reported that the combination of widefield digital imaging and telemedicine was safe and effective in detecting potentially blinding retinopathy of prematurity.

Jackson and colleagues ${ }^{13}$ analyzed the costeffectiveness of telemedicine and digital imaging versus standard examination by ophthalmologists for the management of retinopathy of prematurity. They found that telemedicine was more cost-effective than standard binocular indirect ophthalmoscopy.

\section{What can we expect in the future?}

Guidelines are lacking on the use of telemedicine and digital imaging to screen for retinopathy of prematurity in infants. Until further evidence is available from large, multicentre randomized controlled trials, the combination of telemedicine and digital imaging with a real-time two-way audio-video connection appears to be a useful alternative to binocular indirect ophthalmoscopy for neonatal ICUs where on-site screening services are not available.

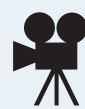

A video showing the use of telemedicine and digital imaging to screen an infant for retinopathy of prematurity is available in Appendix 1, at www.cmaj.ca/lookup/suppl/doi:10.1503/cmaj.131357/-/DC1. 


\section{References}

1. Yogesan K, Goldschmidt L, Cuadros J, editors. Digital teleretinal screening. Teleophthalmology in practice [parts II and III]. New York: Springer-Verlag; 2012.

2. Jefferies A; Canadian Paediatric Society, Fetus and Newborn Committee. Retinopathy of prematurity: recommendations for screening. Paediatr Child Health 2010;15:667-74.

3. Early Treatment for Retinopathy of Prematurity Cooperative Group. Revised indications for the treatment of retinopathy of prematurity: results of the Early Treatment for Retinopathy of Prematurity randomized trial. Arch Ophthalmol 2003;121:1684-94.

4. Seminal Canadian recommendations for evidence-based examinations of neonates for retinopathy of prematurity (SCREEN_ROP). Available: www.screenrop.ca/about_the_study.php (accessed 2014 June 4).

5. Fijalkowski N, Zheng LL, Henderson MT, et al. Stanford University Network for Diagnosis of Retinopathy of Prematurity (SUNDROP): four years of screening with telemedicine. Curr Eye Res 2013;38:283-91.

6. Weaver DT, Murdock TJ. Telemedicine detection of type 1 ROP in a distant neonatal intensive care unit. JAAPOS 2012;16:229-33.

7. Lorenz B, Spasovska K, Elflein H, et al. Wide-field digital imaging based telemedicine for screening for acute retinopathy of prematurity (ROP). Six-year results of a multicentre field study. Graefes Arch Clin Exp Ophthalmol 2009;247:1251-62.

8. Moshfeghi DM. ROP screening using telemedicine. Retina Today 2010(Mar.):59-61.

9. Mehta M, Adams GG, Bunce C, et al. Pilot study of the systemic effects of three different screening methods used for retinopathy of prematurity. Early Hum Dev 2005;81:355-60.

10. Kemper AR, Freedman SF, Wallace DK. Retinopathy of prematurity care: patterns of care and workforce analysis. $J$ AAPOS 2008; $12: 344-8$

11. Vinekar A. Tele-ophthalmology model for ROP management. Ophthalmol Times Eur 2009;5:14-6.

12. Kemper AR, Wallace DK, Quinn GE. Systematic review of digital imaging screening strategies for retinopathy of prematurity. Pediatrics 2008;122:825-30.

13. Jackson KM, Scott KE, Graff-Zivin J, et al. Cost-utility analysis of telemedicine and ophthalmoscopy for retinopathy of prematurity management. Arch Ophthalmol 2008;126:493-9.
Affiliations: Department of Ophthalmology and Vision Sciences (Aziz, Isaac, Tehrani), The Hospital for Sick Children; Department of Ophthalmology and Vision Sciences (Aziz, Tehrani), University of Toronto, Toronto, Ont.

Contributors: Nasrin Najm Tehrani contributed to the conception and design of the study. All of the authors participated in the drafting, critical review and revision of the manuscript, approved the final version submitted for publication and agreed to act as guarantors of the work.

Acknowledgements: The authors thank the Ontario Provincial Council for Maternal and Child Health for its contribution to the set-up of the Ontario Telemedicine for Retinopathy of Prematurity project. They also thank Leslie MacKeen and Cynthia VandenHoven for their contributions to the preparation of the video included with this article.

The Ontario Telemedicine for Retinopathy of Prematurity project received funding from the Ontario Ministry of Health and Long-Term Care.

$C M A J$ invites contributions to Innovations, which highlights recent diagnostic and therapeutic advances. Novel uses of older treatments will also be considered. For publication, the benefits of the innovation, its availability and its limitations must be highlighted clearly, but briefly. Visual elements (images) are essential. Submit brief evidencebased articles (maximum 1000 words and five references) to http://mc.manuscriptcentral .com/cmaj or email diane.kelsall@cmaj.ca to discuss ideas. 